\title{
Electrocardiographic abnormalities and troponin levels provide insight on the prognosis of COVID-19 patients: A single center experience from Iran
}

\author{
Amin Mahdavi ${ }^{\mathbb{D}}$, Meysam Moravej ${ }^{\mathbb{D}}$, Maryam Aliramezany ${ }^{* \mathbb{(}}$ \\ Cardiovascular Research Center, Institute of Basic and Clinical Physiology Sciences, Kerman University of Medical \\ Sciences, Kerman, Iran
}

Received: 23 August 2021 Accepted: 24 November 2021

Published online: 9 December 2021

*Corresponding author: Maryam Aliramezany,

Department of Cardiology,

Afzalipoor Hospital, Kerman, Iran

Email:maliramezany@yahoo.com

Competing interests: None.

Funding information: None.

Citation: Mahdavi A, Moravej M, Aliramezany M. Electrocardiographic abnormalities and troponin levels provide insight on the prognosis of COVID-19 patients: A single center experience from Iran. Journal of Emergency Practice and Trauma 2022; 8(1): 37-42. doi: 10.34172 jept.2021.29.

\begin{abstract}
Objective: Coronavirus disease 2019 (COVID-19) is an infection which can present itself by the involvement of various organs, but the most common manifestations are respiratory symptoms, fever and dyspnea with a high mortality rate. In order to study the prognosis of patients and also to determine the treatment plan, we need non-invasive methods which can be easily used in the triage of patients. In this study, we investigated the diagnostic value of electrocardiographic (ECG) changes and troponin levels in patients with this disease.

Methods: This is a descriptive study. Confirmed COVID-19 patients participated in the present study. Data were collected by taking history and referring to medical records. We analyzed data by using chi square, $t$ test and logistic regression through SPSS software version 22.

Results: One hundred and five patients with COVID-19 disease were examined. Most patients were men $(53.3 \%)$ and the mean age was 54.53 years. The most common underlying diseases were hypertension and diabetes mellitus. Ninety-five patients had abnormal electrocardiography including eleven with long QT; seven with arrhythmia; 78 with sinus tachycardia; 7 with hemi-block; 1 with hemi-block and first degree atrioventricular block; 4 with abnormal axis and 28 with ischemic changes. Eleven patients (10.5\%) had positive troponin level, whose length of hospital stay was higher (12.73 vs. 12.07 days). Furthermore, their mean length of intensive care unit (ICU) stay was also higher. In addition, among the troponin-positive group, $100 \%$ had abnormal electrocardiography.

Conclusion: The findings of the present study showed that ECG abnormalities and troponin levels could provide good information about the prognosis of patients. Moreover, it seems that ECG changes in COVID-19 patients, whether indicative of underlying heart disease or resulted from infection, can affect the prognosis of patients. Therefore, considering ECG findings and troponin levels can help select patients at a higher risk for triage. Keywords: Electrocardiography, Troponin, COVID-19, Myocardial damage
\end{abstract}

Introduction

The global epidemic of coronavirus disease 2019 (COVID-19) has infected millions of people worldwide (1). COVID-19 disease has clinical manifestation due to new corona virus and is often associated with lung involvement that may lead to systemic inflammation, pneumonia and in the severe form of disease, multi-organ damage and acute respiratory distress syndrome.

While most of the focus has been on pulmonary complications, it is also important to be aware of cardiovascular involvement, which can play a significant role in mortality (2).

Involvement of cardiovascular system in patients with COVID-19 infection is accompanied by different myocardial injuries like myocarditis, myocardialinfarction, acute heart failure, arrhythmia and thromboembolic events (3). One of the cardiac complications reported in these patients is acute heart involvement which has been observed in $7.2 \%$ to $12 \%$ of cases and is much higher among critically ill patients (4).

Cardiac involvement in these patients may be reflected by electrocardiographic (ECG) changes and increased troponin level. Based on a previous study, ECG changes usually indicate a serious state in patients infected with COVID-19 and may reflect various heart injuries with a variety of manifestations (5). Additionally, levels of cardiac troponin I (cTnI) increase in patients with severe COVID-19 infection in contrast to those with mild infection (6).

In the first studies reported from Wuhan, China, 
a significant percentage of involved patients (12\%$28 \%)$ showed high cardiac troponin levels with higher troponin levels belonging to older patients and these patients had more associated side effects, including high blood pressure, ischemic heart disease, and diabetes mellitus (7). Higher troponin levels in infected patients were more likely associated with admission to the intensive care unit (ICU) and patients had higher hospital mortality rates. In addition, acute respiratory infections are usually associated with high troponin levels, which can be used as an indicator of the disease severity and can predict upcoming cardiac events (8). It is important to mention that the association of COVID-19 with heart disease can both increase the risk of infection and worsen the prognosis. In this regard, the ECG can be used as an essential tool for monitoring of cardiac condition and understanding cardiovascular manifestations of COVID-19 (1). However, manifestations of the disease during acute phase and its effects on ECG are not well defined yet (9).

In the present research, we aimed to define the initial changes of ECG and troponin levels in COVID-19 patients and their association with the disease prognosis.

\section{Methods}

This descriptive analytical study was performed on 105 confirmed cases of COVID-19 referred to Afzalipour Hospital in Kerman, Iran from Feb 2020 to June 2020. All participants completed the written informed consent and study protocol was approved by the Ethics Committee of Research Deputy of Kerman University of Medical Sciences (ID: IR.KMU.AH.REC.1399.87). Infection with new corona virus was confirmed by a pharyngeal swap test (10). We excluded Patients with implanted cardiac pacemaker from the study as the ECG could not be evaluated.

At admission time, a complete history was taken and a full examination was performed. Medical and habitual history were taken during the patient examination and completed by referring to medical records.

Blood samples were taken from patients to perform necessary tests including biochemistry, liver and coagulations tests and troponin levels. Troponin levels larger or equal to $0.3 \mathrm{ng} / \mathrm{mL}$ were considered positive.

ECG was taken with a standard calibrated device. According to the study definition, in patients with any changes regarding the clinical condition or laboratories tests, ECG was repeated. Then, ECG tracings were analyzed by two cardiologists participating in the study.

The parameters that measured the electrocardiogram included heart rate, corrected QT interval, the presence of ST-T abnormalities (including ST depression or elevation, inverted T), axis deviation and the presence of arrhythmia and heart block.

Interval between the initiation of the $\mathrm{Q}$ wave to the end of the T wave was assumed QT interval and corrected by Bazett's formula according to the heart rate (9). After that, PR interval and QRS duration were measured.

Criteria for ST-T changes considered in this study included one of the followings: (a) flat or biphasic T-wave amplitude with $<0.10 \mathrm{mV}$ in lead Avl; I, II or V3-V6; (b) ST depression $\geq 0.05 \mathrm{mV}$, in any leads aVL, I, II or V3-V6 coexistence with T-wave inversion; (c) depression of ST $\geq 0.10 \mathrm{mV}$ and upward sloping of ST-segment, in any of leads Avl, V2- V6 or I, II; (d) depression of ST-J > $0.05 \mathrm{mV}$ below P-R baseline was seen in one of the leads including Avl, V2-V6 or I, II (9); (e): ST elevation at least $0.1 \mathrm{mV}$ in a limb lead or $0.2 \mathrm{mV}$ in a precordial lead (at point 0.04 seconds after the J-point). The baseline is either the TP interval or PR interval (11).

We gave a code and completed a pre-designed form comprising ECG, demographics, medical history and laboratory tests data for each patient. All data including early vital signs, demographic information (age and gender); medical and habitual history as well as morbidity information to the current disease, including the initial manifestation of COVID-19 disease and length of stay in the ICU and medications received for COVID-19 disease and laboratory information were manually extracted from the hospital information system. Finally, the patient's survival or death was recorded.

All data were encoded and entered into SPSS software (version 22). Mean, standard deviation, frequency and percentage were used to describe the data. Independent samples $t$ test and chi-square were used for analysis of data. A $P$ value equal or less than 0.05 was considered statistically significant.

\section{Results}

In this study, 105 patients with COVID-19 infection were examined. Most patients were men (53.3\%) and the mean age was 54.53 years. The most common underlying diseases were hypertension and diabetes mellitus. Ninetyfive patients had abnormal ECG of which eleven had long QT, and seven had arrhythmia (two with atrial fibrillations, one with premature atrial complex, and four with premature ventricular complex). Seventy-eight patients had sinus tachycardia, seven had hemi-block, one had hemi-block and first degree atrio-ventricular block, four had abnormal axis and twenty-eight had ischemic changes (four patients had ST elevation myocardial infarction (STEMI), and twenty-four had inverted $\mathrm{T}$ and ST depression).

Unfortunately, fourteen patients died during hospitalization, of which eleven had an underlying disease (Five had hypertension and six had diabetes mellitus) and there was a significant difference $(P$ value $\leq 0.05)$ between died and survived cases in terms of fever, ICU admission, and underlying diseases (see Table 1).

Among died patients, one patient had long QT; two had 
Table 1. Comparison of demographics and laboratory findings by prognosis and ECG findings

\begin{tabular}{|c|c|c|c|c|c|c|c|}
\hline & & Died & Survived & $P$ value & Normal EKG & Abnormal EKG & $P$ value \\
\hline \multirow{2}{*}{ Gender, No. (\%) } & Male & $6(10.7)$ & $50(89.3)$ & \multirow{2}{*}{0.39} & $5(8.9)$ & $51(91.1)$ & \multirow{2}{*}{0.82} \\
\hline & Female & $8(16.3)$ & $41(83.7)$ & & $5(10.2)$ & $44(89.8)$ & \\
\hline \multirow{2}{*}{ Addiction } & Yes & $3(20)$ & $12(80)$ & \multirow{2}{*}{0.41} & $1(6.7)$ & $14(93.3)$ & \multirow{2}{*}{0.68} \\
\hline & No & $11(12.2)$ & 79 (87.8) & & $9(10)$ & $81(90)$ & \\
\hline \multirow{3}{*}{ Primary presentations } & Fever & $5(6.8)$ & $68(93.2)$ & 0.00 & $4(5.5)$ & $69(94.5)$ & 0.03 \\
\hline & Cough & $6(14.3)$ & $36(85.7)$ & 0.81 & $2(4.8)$ & $40(95.2)$ & 0.17 \\
\hline & Dyspnea & $11(15.9)$ & $58(84.1)$ & 0.27 & $7(10.1)$ & $62(89.9)$ & 0.76 \\
\hline \multirow{2}{*}{ ICU admission } & Yes & $1(1.5 \%)$ & $67(98.5 \%)$ & \multirow{2}{*}{0.00} & $4(10.8))$ & $33(89.2)$ & \multirow{2}{*}{0.74} \\
\hline & No & $13(35.1)$ & $24(64.9)$ & & $6(8.8)$ & $62(91.2)$ & \\
\hline Mean age (Mean \pm SD) & & $51.79 \pm 5.34$ & $54.96 \pm 1.88$ & 0.54 & $62.30 \pm 5.14$ & $53.72 \pm 1.87$ & 0.15 \\
\hline Mean length of hospitalization (Mean \pm SD) & & $13.21 \pm 2.76$ & $11.98 \pm 0.7$ & 0.55 & $11.80 \pm 3.55$ & $12.18 \pm 0.69$ & 0.87 \\
\hline Mean ICU stay $($ Mean \pm SD) & & $9.43 \pm 2.85$ & $3.34 \pm 0.68$ & 0.00 & $4.70 \pm 2.41$ & $4.09 \pm 0.76$ & 0.80 \\
\hline Systolic blood pressure (Mean \pm SD) & & $124.28 \pm 6.53$ & $128.04 \pm 2.14$ & 0.53 & $125.10 \pm 5.29$ & $127.8 \pm 2.19$ & 0.70 \\
\hline Diastolic blood pressure (Mean \pm SD) & & $78.14 \pm 4.15$ & $78.51 \pm 1.38$ & 0.92 & $75.8 \pm 4.05$ & $78.74 \pm 1.39$ & 0.51 \\
\hline Heart rate beat per minute (Mean \pm SD) & & $96.64 \pm 4.68$ & $101.43 \pm 1.5$ & 0.26 & $82.20 \pm 3.48$ & $102.75 \pm 1.41$ & 0.00 \\
\hline Blood urea nitrogen (Mean \pm SD) & & $45.5 \pm 5.8$ & $43.05 \pm 4.2$ & 0.82 & $74.00 \pm 3.25$ & $40.16 \pm 3.20$ & 0.00 \\
\hline Serum creatinine concentration, (Mean \pm SD) & & $1.07 \pm 0.19$ & $1.33 \pm 0.14$ & 0.49 & $1.5 \pm 0.42$ & $1.27 \pm 0.13$ & 0.60 \\
\hline Alanine aminotransferase (Mean \pm SD) & & $29.21 \pm 3.55$ & $68.48 \pm 35.87$ & 0.67 & $37.00 \pm 6.95$ & $66.01 \pm 34.37$ & 0.78 \\
\hline Aspartate aminotransferase (Mean \pm SD) & & $37.93 \pm 5.5$ & $60.29 \pm 20.63$ & 0.67 & $35.8 \pm 6.53$ & $59.57 \pm 19.56$ & 0.69 \\
\hline Alkaline phosphatase (Mean \pm SD) & & $248.2 \pm 21.79$ & $208.95 \pm 8.84$ & 0.10 & $231.30 \pm 30.46$ & $212.38 \pm 8.58$ & 0.50 \\
\hline Prothrombin time (Mean \pm SD) & & $15.36 \pm 0.82$ & $14.79 \pm 0.33$ & 0.54 & $14.10 \pm 0.31$ & $14.95 \pm 0.34$ & 0.42 \\
\hline International normalized ratio (Mean \pm SD) & & $1.29 \pm 0.12$ & $1.19 \pm 0.05$ & 0.52 & $1.00 \pm 0.00$ & $1.22 \pm 0.005$ & 0.00 \\
\hline Partial thromboplastin time $($ Mean \pm SD) & & $40.21 \pm 4.13$ & $36.19 \pm 1.11$ & 0.21 & $40.30 \pm 5.56$ & $36.35 \pm 1.08$ & 0.50 \\
\hline Troponin $($ Mean \pm SD) & & $0.52 \pm 0.32$ & $0.35 \pm 0.16$ & 0.70 & $0.1 \pm 0.0001$ & $0.41 \pm 0.16$ & 0.55 \\
\hline
\end{tabular}

arrhythmia (atrial fibrillation and premature ventricular complex); eight had sinus tachycardia; seven had ischemic changes (one patient had STEMI and six had inverted $\mathrm{T}$ and ST depression) and one had abnormal axis. Only ischemic changes in the died and survived groups were statistically significant $(P$ value $=0.01)$ (see Table 2$)$.

Also, eleven confirmed COVID-19 cases (10.5\%) had troponin above 0.3 , of which most were males. The mean age of patients with troponin positive was higher (56.36 years compared to 54.32 years) and nine of them were not addicted and all eleven patients had underlying diseases (four with hypertension; one with lung disease; two with ischemic heart disease; one with hyperthyroidism; one with hyperlipidemia and two with diabetes mellitus). The length of hospital (12.73 vs. 12.07 days) and ICU stay was higher in patients that had positive troponin. Only the mean blood pressure in patients with positive and negative troponin levels was statistically different $(P$ value $=0.01)$. Based on our statistical analysis, hospitalization in the ICU $(P$ value $=0.00)$ and taking remdesivir $(P$ value $=0.02)$ and also the mean blood pressure $(P$ value $=0.00)$ in patients with troponin levels were significantly different in positive and negative troponin groups.

Among patients with positive troponin (11 cases),
$100 \%$ had abnormal ECGs, of which two had long QT and eight had sinus tachycardia; four had STEMI; ten had inverted $\mathrm{T}$ and ST depression and only ischemic changes were significant in terms of troponin $(P$ value $=0.00)$ (see Table 3).

\section{Discussion}

In this study, we used ECG changes and troponin levels as non-invasive screening methods to evaluate the prognosis and cardiac complications in patients with confirmed COVID-19 disease.

Our findings show that the most common ECG changes in hospitalized patients were sinus tachycardia and ST-T changes. In addition, the commonest ECG changes observed in patients admitted to the ICU were sinus tachycardia. These results are somehow similar to the findings obtained by McCullough et al expressing that the most common changes in the ECG of COVID-19 infected patients were the presence of inverted $\mathrm{T}$ and nonspecific repolarization abnormalities (12).

In general, the presence of an abnormal ECG may indicate the underlying cardiovascular disease (CVD) or can be the result of COVID-19 infection effects (direct or indirect) on the cardiovascular system. Overall, the 
Table 2. Comparison of ECG parameters in died and survived cases

\begin{tabular}{|c|c|c|c|c|}
\hline & & Died & Survived & $P$ value \\
\hline \multirow{2}{*}{ Normal ECG } & Yes & $3(30)$ & $7(70)$ & \multirow{2}{*}{0.10} \\
\hline & No & $11(11.06)$ & $84(88.4)$ & \\
\hline \multirow{2}{*}{ Long QT } & Yes & $1(9.1)$ & $10(90.9)$ & \multirow{2}{*}{0.66} \\
\hline & No & $13(13.8)$ & $81(86.2)$ & \\
\hline \multirow{4}{*}{ Arrhythmia } & $\mathrm{AF}$ & $1(50)$ & $1(50)$ & \multirow{4}{*}{0.38} \\
\hline & PAC & $0(0)$ & $1(100)$ & \\
\hline & PVC & $1(25)$ & $3(75)$ & \\
\hline & No & $12(12.2)$ & $86(87.8)$ & \\
\hline \multirow{2}{*}{ Sinus tachycardia } & Yes & $8(10.3)$ & $70(89.7)$ & \multirow{2}{*}{0.11} \\
\hline & No & $6(22.2)$ & $21(77.8)$ & \\
\hline \multirow{2}{*}{$\begin{array}{l}\text { Atrio-ventricular } \\
\text { block }\end{array}$} & No & $0(0)$ & $7(100)$ & \multirow{2}{*}{0.51} \\
\hline & Yes & $14(14.3)$ & $84(85.7)$ & \\
\hline \multirow{2}{*}{ Normal axis } & Yes & $13(12.9)$ & $88(87.1)$ & \multirow{2}{*}{0.48} \\
\hline & No & $1(25)$ & $3(75)$ & \\
\hline \multirow{3}{*}{ Ischemic changes } & STEMI & $1(25)$ & $3(75)$ & \multirow{3}{*}{0.01} \\
\hline & $\begin{array}{l}\text { Inverted T/ST } \\
\text { depression }\end{array}$ & $6(25)$ & $18(75)$ & \\
\hline & No & 7 (9.09) & 70 (90.91) & \\
\hline
\end{tabular}

AF: atrial fibrillation; PAC: premature atrial complex; PVC: premature ventricular complex; STEMI:ST-elevation myocardial infarction.

current study showed that the presence of abnormal ECG in COVID-19 patients at the time of admission is associated with an increase in hospital length of stay and mortality rate, however, the difference was not statistically significant. One reason for this may be that an abnormal
ECG may indicate ischemia, ventricular dysfunction, or underlying heart disease. In addition, the available evidence suggests that myocardial infarction, myocarditis, and acute coronary syndrome are the most commonly observed disorders following COVID-19 infection (13) that could affect prognosis.

Another finding in our study was cardiac arrhythmias that can result from excessive secretion of inflammatory cytokines which is a common result in COVID-19 infected patients. Furthermore, cardiac arrhythmias are usually associated with elevation in the left heart chamber filling pressures, particularly following acute myocardial infarction (14).

Our patients experienced cardiac arrhythmias as well as deviation of axis, likewise, the study conducted by McCullough et al, showed that the presence of cardiac arrhythmias (due to left or right heart involvement) is accompanied by an increased death risk (12). However, in this study, there was no association between arrhythmia and rate of mortality, which may be due to the smaller sample size.

In addition to ECG changes, heart damage in these patients can manifest itself as an increase in the level of the cardiac troponin (5). In patients with the severe form of SARS-CoV-2 infection, cTnI levels increase compared with those with mild infection (15). By evaluating the data first reported from China, a significant percentage of patients (12\%-28\%) revealed a high level of cardiac troponin. People with higher troponin levels were older than patients with normal levels and clearly had higher

Table 3. Comparison of ECG parameters in patients with elevated and normal troponin level

\begin{tabular}{|c|c|c|c|c|}
\hline & & Frequency $(\%)<0.3$ & Frequency $(\%) \geq 0.3$ & $P$ value \\
\hline \multirow{2}{*}{ Normal ECG } & Yes & $10(10.6)$ & $0(0)$ & \multirow{2}{*}{0.25} \\
\hline & No & $84(89.4)$ & $11(100)$ & \\
\hline \multirow{2}{*}{ Long QT } & Yes & $10(10.6)$ & $2(18.2)$ & \multirow{2}{*}{0.37} \\
\hline & No & $84(89.4)$ & 9 (81.8) & \\
\hline \multirow{4}{*}{ Arrhythmia } & $\mathrm{AF}$ & $2(2.1)$ & $0(0)$ & \multirow{4}{*}{0.83} \\
\hline & PAC & $1(1.1)$ & $0(0)$ & \\
\hline & PVC & $4(4.3)$ & $0(0)$ & \\
\hline & No & $87(92.6)$ & $11(100)$ & \\
\hline \multirow{2}{*}{ Sinus tachycardia } & Yes & $70(86.1)$ & $8(72.7)$ & \multirow{2}{*}{0.90} \\
\hline & No & $24(25.5)$ & $3(27.3)$ & \\
\hline \multirow{3}{*}{ Atrio-ventricular block } & No & $87(92.6)$ & $10(90.9)$ & \multirow{3}{*}{0.89} \\
\hline & Hemi-block & $14(13.9)$ & $84(85.7)$ & \\
\hline & Hemi-block/first degree AV block & & & \\
\hline \multirow{2}{*}{ Normal axis } & Yes & $90(95.7)$ & $11(100)$ & \multirow{2}{*}{0.48} \\
\hline & No & $4(4.3)$ & $0(0)$ & \\
\hline \multirow{3}{*}{ Ischemic changes } & STE MI & $0(0)$ & $4(36.4)$ & \multirow{3}{*}{0.00} \\
\hline & Inverted T/ST depression & $19(20.2)$ & $5(45.5)$ & \\
\hline & No & $75(79.8)$ & $2(18.1)$ & \\
\hline
\end{tabular}

AF: atrial fibrillation; PAC: premature atrial complex; PVC: premature ventricular complex; STEMI:ST-elevation myocardial infarction. 
rates of association with other underlying conditions, including high blood pressure, coronary artery disease, and diabetes mellitus. Patients with a higher level of troponin were significantly more likely to be admitted to the ICU with a higher rate of mortality in hospital (8).

Furthermore, acute respiratory infections are usually accompanied by high troponin levels, which may be used as a sign of the severity of the disease and to predict future cardiovascular events. In general, it can be concluded that troponin is a useful indicator of prognosis and progression in patients with COVID-19 infection. Several mechanisms, including viral myocarditis, myocardial damage due to cytokines, micro-angiopathy and latent coronary artery disease may explain this issue (13).

In a meta-analysis of data from four main articles, which included data from 341 patients, it was found that the mean serum level of cardiac troponin in patients with the severe form of COVID-19 infection was significantly higher than non-severe form of the disease (16). The results of our study are in line with these findings and showed that all patients (100\%) with positive troponin had abnormal electrocardiography.

Guo et al in a study on 187 confirmed COVID-19 patients showed that 66 patients $(35.3 \%)$ had CVD, including hypertension, cardiomyopathy and coronary heart disease and 52 patients (27.8\%) showed damage of myocardium, which was specified by an increase in TnT levels. In-hospital mortality of patients without baseline CVD and normal troponin levels were $7.62 \%$ (8 out of $105)$, and for patients with underlying CVD and normal TnT levels, the percentage was $13.33 \%$ (4 of 30) and $37.50 \%$ (6 of 16 ), respectively (14).

In addition, in the study conducted by Shi et al, an overall of 82 patients (19.7\%) with heart damage required more non-invasive mechanical ventilation and had complications including acute respiratory distress syndrome, renal and electrolyte disorders. The prevalence of death and mortality was higher in this group of patients (17).

In our study, the commonest underlying disease was hypertension $(n=8)$ followed by diabetes mellitus $(\mathrm{n}=30)$, which are similar to the study conducted by Li et al. In their study, they reported the ratio of hypertension, CVD and diabetes mellitus in patients with COVID-19 at $17.1 \%, 16.4 \%$ and $9.7 \%$, respectively. The incidence of hypertension, CVD and diabetes mellitus in patients admitted to the ICU doubled, tripled and doubled, respectively compared to non-ICU patients. In addition, the incidence of acute heart damage in patients admitted to the ICU was about 13 times higher than non-hospitalized patients in the ICU (18).

In the present study, diabetes mellitus had no effect on patient mortality, which is similar to the results of the study conducted by Zhou et al that evaluated 191 confirmed COVID-19 patients (7). However, a study by Pishgahi et al showed that diabetes mellitus is a predictor of mortality in patients with COVID-19 disease (19). The reason for this difference could be the retrospective design of the study by Zhou et al and the difference between the populations of Iran and China. In another study that was conducted on 1590 patients, a significant association was found between diabetes mellitus and adverse prognosis in patients with COVID-19 (20).

One of the possible mechanisms suggested for the relationship between COVID-19 and diabetes mellitus is the interaction of the virus with the angiotensinconverting enzyme, which is expressed in vascular and epithelial cells. Diabetes mellitus appears to increase the expression of proteins in this pathway, which worsens the patient's condition (7). However, this proposed mechanism has recently been challenged and questioned (6). Another mechanism that can be considered to aggravate the condition of COVID-19 patients for diabetes mellitus is its association with pulmonary embolism as diabetes mellitus is one of the known risk factors for pulmonary embolism (21,22).

Another important point to note about ECG changes in patients with COVID-19 is the QTc interval. We know that one of the side effects of common medications used for treating of these patients, (hydroxychloroquine and some antiviral drugs), is an increase in QTc interval. Because this change in ECG can lead to fatal arrhythmias such as torsade de pointes and ventricular tachycardia, patients, especially those hospitalized, should be monitored continuously.

Overall, the present study is one of the few observational studies on changes in ECG and level of troponin in COVID-19 patients. The obtained results are unswerving with emerging data indicating that cardiac involvement is an imperative indicator of prognosis in COVID-19. Furthermore, the study shows that monitoring of patients with ECG and troponin levels can be important in determining the triage of patients for transmission to ICU, method of treatment and prognosis of them.

\section{Conclusion}

To sum up, ECG changes and troponin levels could provide good information about the prognosis of patients for the medical team. It seems that changes of ECG in COVID-19 infected patients, whether indicative of underlying heart disease or resulted from infection, can affect the prognosis of patients. Therefore, considering ECG findings and troponin levels can help to identify patients at a higher risk when we are not sure about patient management as an inpatient or outpatient, and when triage is needed to allocate equipment to patients at higher or more urgent needs.

\section{Limitations}

Comparatively small sample size is one of the limitations of the present study. However, data and clinical results were manually extracted from the electronic records using 
a method that had high reliability. On the other hand, all ECGs were interpreted by two experienced electrocardiologists, both of which can increase the accuracy of the study. Given that we did not have a previous ECG in all of them, we could not compare the preceding ECG with the present ECG changes, and this aspect must be considered in the design of future studies.

\section{Availability of data and material}

The datasets of the current study are not publicly available but are available from the corresponding author upon request.

\section{Authors' contributions}

MA and AM managed the patients, drafted the paper; MM drafted the paper. All authors read and approved the manuscript.

\section{Ethics issues}

Our study design and protocols were accepted by the Ethics Committee of Research Deputy of Kerman University of Medical Sciences (ID: IR.KMU.AH.REC.1399.87) and all participants completed the written informed consent form at the beginning of the study. They were also free to leave the study at any stage of the study.

\section{Acknowledgements}

We thank our kind patients and our respectful colleagues who made this research possible.

\section{References}

1. Moazenzadeh M, Jafari F, Farrokhnia M, Aliramezany M. First reported case of unrepaired tetralogy of Fallot complicated with coronavirus disease-19 (COVID-19). Cardiol Young 2020; 30(9): 1339-42. doi: 10.1017/ s1047951120001821.

2. Long B, Brady WJ, Koyfman A, Gottlieb M. Cardiovascular complications in COVID-19. Am J Emerg Med 2020; 38(7): 1504-7. doi: 10.1016/j.ajem.2020.04.048.

3. Bandyopadhyay D, Akhtar T, Hajra A, Gupta M, Das A, Chakraborty S, et al. COVID-19 pandemic: cardiovascular complications and future implications. Am J Cardiovasc Drugs 2020; 20(4): 311-24. doi: 10.1007/s40256-02000420-2.

4. He J, Wu B, Chen Y, Tang J, Liu Q, Zhou S, et al. Characteristic electrocardiographic manifestations in patients with COVID-19. Can J Cardiol 2020; 36(6): 966. e1-966.e4. doi: 10.1016/j.cjca.2020.03.028.

5. Huang C, Wang Y, Li X, Ren L, Zhao J, Hu Y, et al. Clinical features of patients infected with 2019 novel coronavirus in Wuhan, China. Lancet 2020; 395(10223): 497-506. doi: 10.1016/s0140-6736(20)30183-5.

6. Wang D, Hu B, Hu C, Zhu F, Liu X, Zhang J, et al. Clinical characteristics of 138 hospitalized patients with 2019 novel coronavirus-infected pneumonia in Wuhan, China. JAMA 2020; 323(11): 1061-9. doi: 10.1001/jama.2020.1585.

7. Zhou F, Yu T, Du R, Fan G, Liu Y, Liu Z, et al. Clinical course and risk factors for mortality of adult inpatients with COVID-19 in Wuhan, China: a retrospective cohort study. Lancet 2020; 395(10229): 1054-62. doi: 10.1016/s01406736(20)30566-3.
8. Tersalvi G, Vicenzi M, Calabretta D, Biasco L, Pedrazzini G, Winterton D. Elevated troponin in patients with coronavirus disease 2019: possible mechanisms. J Card Fail 2020; 26(6): 470-5. doi: 10.1016/j.cardfail.2020.04.009.

9. Angeli F, Spanevello A, De Ponti R, Visca D, Marazzato J, Palmiotto G, et al. Electrocardiographic features of patients with COVID-19 pneumonia. Eur J Intern Med 2020; 78: 101-6. doi: 10.1016/j.ejim.2020.06.015.

10. Yang Z, Shi J, He Z, Lü Y, Xu Q, Ye C, et al. Predictors for imaging progression on chest $\mathrm{CT}$ from coronavirus disease 2019 (COVID-19) patients. Aging (Albany NY) 2020; 12(7): 6037-48. doi: 10.18632/aging.102999.

11. Mann D, Zipes D, Libby P, Bonow R. Braunwalds Heart Disease: A Textbook of Cardiovascular Medicine. Saunders: Elsevier; 2015.

12. McCullough SA, Goyal P, Krishnan U, Choi JJ, Safford MM, Okin PM. Electrocardiographic findings in coronavirus disease-19: insights on mortality and underlying myocardial processes. J Card Fail 2020; 26(7): 626-32. doi: 10.1016/j. cardfail.2020.06.005.

13. Driggin E, Madhavan MV, Bikdeli B, Chuich T, Laracy J, Biondi-Zoccai G, et al. Cardiovascular considerations for patients, health care workers, and health systems during the COVID-19 pandemic. J Am Coll Cardiol 2020; 75(18): 2352-71. doi: 10.1016/j.jacc.2020.03.031.

14. Guo T, Fan Y, Chen M, Wu X, Zhang L, He T, et al. Cardiovascular implications of fatal outcomes of patients with coronavirus disease 2019 (COVID-19). JAMA Cardiol 2020; 5(7): 811-8. doi: 10.1001/jamacardio.2020.1017.

15. Wan Y, Shang J, Graham R, Baric RS, Li F. Receptor recognition by the novel coronavirus from Wuhan: an analysis based on decade-long structural studies of SARS coronavirus. J Virol 2020; 94(7): e00127-20. doi: 10.1128/ jvi.00127-20.

16. Lippi G, Lavie CJ, Sanchis-Gomar F. Cardiac troponin I in patients with coronavirus disease 2019 (COVID-19): evidence from a meta-analysis. Prog Cardiovasc Dis 2020; 63(3): 390-1. doi: 10.1016/j.pcad.2020.03.001.

17. Shi S, Qin M, Shen B, Cai Y, Liu T, Yang F, et al. Association of cardiac injury with mortality in hospitalized patients with COVID-19 in Wuhan, China. JAMA Cardiol 2020; 5(7): 802-10. doi: 10.1001/jamacardio.2020.0950.

18. Li B, Yang J, Zhao F, Zhi L, Wang X, Liu L, et al. Prevalence and impact of cardiovascular metabolic diseases on COVID-19 in China. Clin Res Cardiol 2020; 109(5): 531-8. doi: 10.1007/s00392-020-01626-9.

19. Pishgahi M, Yousefifard M, Safari S, Ghorbanpouryami F. Electrocardiographic findings of COVID-19 patients and their correlation with outcome; a prospective cohort study. Front Emerg Med 2020; 5(2): e17. doi: 10.18502/fem. v5i2.5608.

20. Zhou P, Yang XL, Wang XG, Hu B, Zhang L, Zhang W, et al. A pneumonia outbreak associated with a new coronavirus of probable bat origin. Nature 2020; 579(7798): 270-3. doi: 10.1038/s41586-020-2012-7.

21. Fang L, Karakiulakis G, Roth M. Are patients with hypertension and diabetes mellitus at increased risk for COVID-19 infection? Lancet Respir Med 2020; 8(4): e21. doi: 10.1016/s2213-2600(20)30116-8.

22. Khajali Z, Sanati HR, Pouraliakbar H, Mohebbi B, Aeinfar K, Zolfaghari R. Self-expandable stent for repairing coarctation of the left-circumferential aortic arch with right-sided descending aorta and aberrant right subclavian artery with Kommerell's aneurysm. Ann Vasc Surg 2017; 38: 318.e7-318.e10. doi: 10.1016/j.avsg.2016.05.116. 